\title{
TFE3, HSP47, and CREB3 Pathways of the Mammalian Golgi Stress Response
}

\author{
Mai Taniguchi, and Hiderou Yoshida* \\ Department of Molecular Biochemistry, Graduate School of Life Science, University of Hyogo, Hyogo \\ 678-1297, Japan
}

\begin{abstract}
The capacity of each organelle in eukaryotic cells is tightly regulated in accordance with cellular demands by specific regulatory systems, which are generically termed organelle autoregulation. The Golgi stress response is one of the systems of organelle autoregulation and it augments the capacity of Golgi function if this becomes insufficient (Golgi stress). Recently, several pathways of the mammalian Golgi stress response have been identified, specifically the TFE3, HSP47, and CREB3 pathways. This review summarizes the essential parts of the Golgi stress response from the perspective of the organelle autoregulation.
\end{abstract}

Key words: TFE3, MLX, HSP47, CREB3, organelle autoregulation

\section{Introduction}

Eukaryotic cells contain various subcellular compartments that are specialized with specific functions, which are called organelles. Organelles in mammalian cells include the nucleus, mitochondria, endoplasmic reticulum (ER), Golgi apparatus, lysosomes, and peroxisomes. The amount of each organelle is tightly regulated in accordance with cellular demands by mechanisms termed organelle autoregulation (Sasaki and Yoshida, 2015) (Fig. 1). For instance, the amount of the ER is remarkably increased during the differentiation of plasma cells by the mechanism called the ER stress response or the unfolded protein response because the function of the ER becomes insufficient (ER stress) due to the synthesis of large amounts of immunoglobulins (Yoshida, 2007). Similarly, the lysosome stress response upregulates lysosomal function when lysosomal function becomes insufficient (lysosome stress) and substrates for lysosomal hydrolases accumulate in the lysosomes (Napolitano and Ballabio, 2016). In addition, the mitochondrial unfolded protein response increases a set of mitochondrial proteins (mitochondrial chaperones and proteases) when unfolded proteins accumulate in the mitochondrial matrix and cells suffer from insufficiency of mitochondrial function (mitochondrial stress) (Lin and Haynes, 2016). Finally, the amount of peroxisomes is augmented by transcription factor PPAR $\alpha$ when long chain

\footnotetext{
*To whom correspondence should be addressed: Hiderou Yoshida, Department of Molecular Biochemistry, Graduate School of Life Science, University of Hyogo, Harima Science Garden City, Hyogo 678-1297, Japan.

Tel: +81-791-58-0212, Fax: +81-791-58-0219

E-mail: hide@sci.u-hyogo.ac.jp

Web site: http://www.sci.u-hyogo.ac.jp/life/biochem2/index-j.html
}

fatty acids, substrates for peroxisomal metabolic enzymes, accumulate in cells (Chakravarthy et al., 2009). Thus, organelle autoregulation is an indispensable mechanism for eukaryotic cells to automatically maintain homeostasis of each organelle, which is a fundamental issue in cell biology.

Although the molecular mechanisms of organelle autoregulation seem diverse and complicated, the essential mechanisms consists from three key components, namely, a sensor that detects insufficiency of organelle function, a transcription factor that increases transcription of genes involved in organelle function, and an enhancer element to which the transcription factor binds. In the case of the mammalian ER stress response, there are three response pathways. The sensor, transcription factor, and enhancer of the ATF6 pathway are pATF6(P), pATF6(N), and ERSE, respectively, for the IRE1 pathway they are IRE1, pXBP1(S), and UPRE, and those of the PERK pathway are PERK, ATF4, and AARE (Gardner et al., 2013; Kimata and Kohno, 2011; Mori, 2015; Volmer and Ron, 2015) (Fig. 2).

The Golgi stress response is an autoregulatory system of the Golgi apparatus, which augments Golgi function when it becomes insufficient (Golgi stress). For instance, the Golgi apparatus is remarkably increased in mucous cells in the Brunner's glands, which secrete large amounts of mucins (Berger and Roth, 1997). Because mucins are highly glycosylated in the Golgi, the capacity of the Golgi becomes insufficient during the differentiation of mucous cells, and these cells augment the Golgi apparatus using the Golgi stress response. The molecular mechanism of the Golgi stress response has been less analyzed compared with that of the ER stress response, but recent studies revealed an important part of the regulatory mechanism, including the TFE3, HSP47, and CREB3 pathways (Fig. 3) (Miyata 


\section{Organelle Autoregulation}
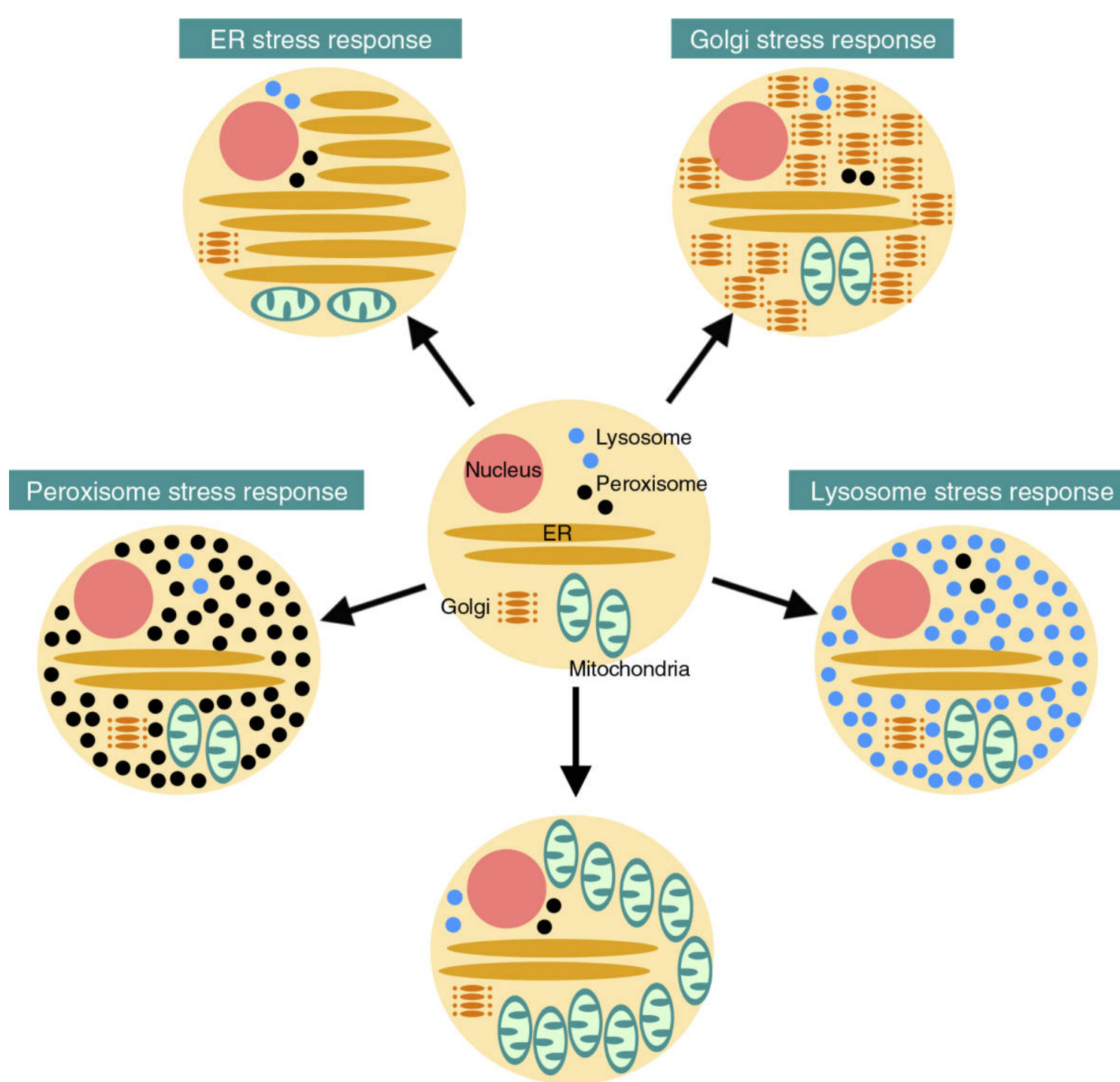

Mitochondria stress response

Fig. 1. Organelle autoregulation. The capacity of each organelle (ER, Golgi, lysosome, mitochondria, peroxisome, and nucleus) is tightly regulated in accordance with cellular demands by organelle autoregulation (ER stress response, Golgi stress response, lysosome stress response, and mitochondria stress response [which is also called the mitochondrial unfolded protein response], peroxisome stress response, and cell cycle).

et al., 2013; Oku et al., 2011; Reiling et al., 2013; Taniguchi et al., 2015, 2016).

\section{TFE3 pathway}

\section{(1) Golgi stress inducers for the TFE3 pathway}

In order to analyze the molecular mechanism of the Golgi stress response, chemical reagents or genetic mutations that artificially activate the Golgi stress response in experimental systems are indispensable. In the case of the ER stress response, chemicals such as thapsigargin, tunicamycin, and dithiothreitol are used as ER stress inducers (Yoshida, 2007). Thapsigargin is an inhibitor of ER-localized $\mathrm{Ca}^{2+}-$ ATPase (SERCA) that transports $\mathrm{Ca}^{2+}$ ions from the cytosol to the ER. Because $\mathrm{Ca}^{2+}$ ions are indispensable for the activity of ER chaperones, thapsigargin treatment markedly 


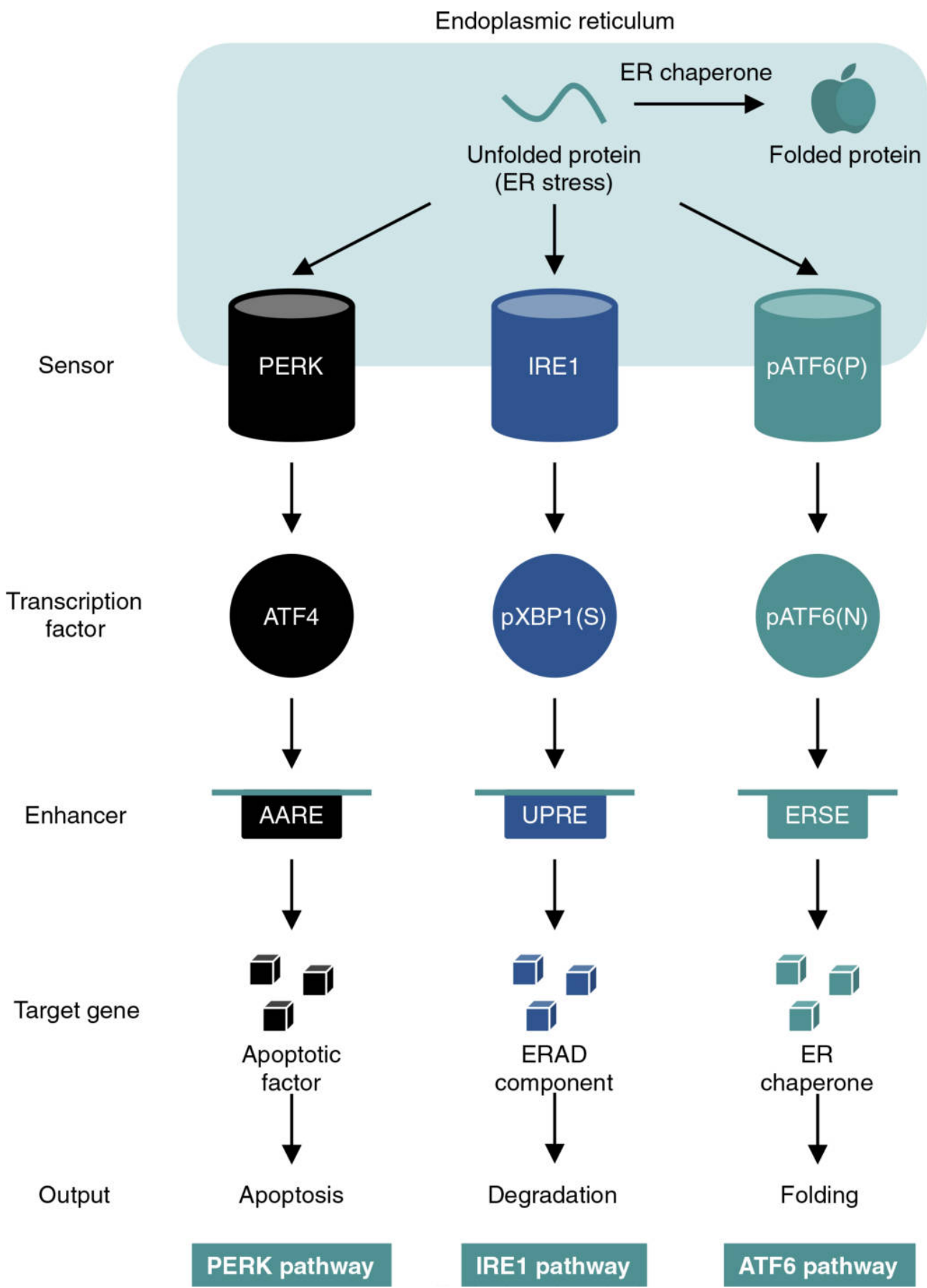

Fig. 2. The mechanism of the mammalian ER stress response. Secretory and membrane proteins are synthesized in the ER and folded with the assistance of ER chaperones. Malfolded proteins are degraded by the ERAD. When the synthesis of secretory and membrane proteins increases and overwhelms the capacity of the ER, they accumulate in the ER as unfolded proteins. Accumulation of unfolded proteins in the ER is detected by sensor molecules (PERK, IRE1, and pATF6(P)) as ER stress, and this activates specific transcription factors (ATF4, pXBP1(S), and pATF6(N)), resulting in the transcriptional induction of ER-related genes through binding to specific enhancers (AARE, UPRE, and ERSE). 


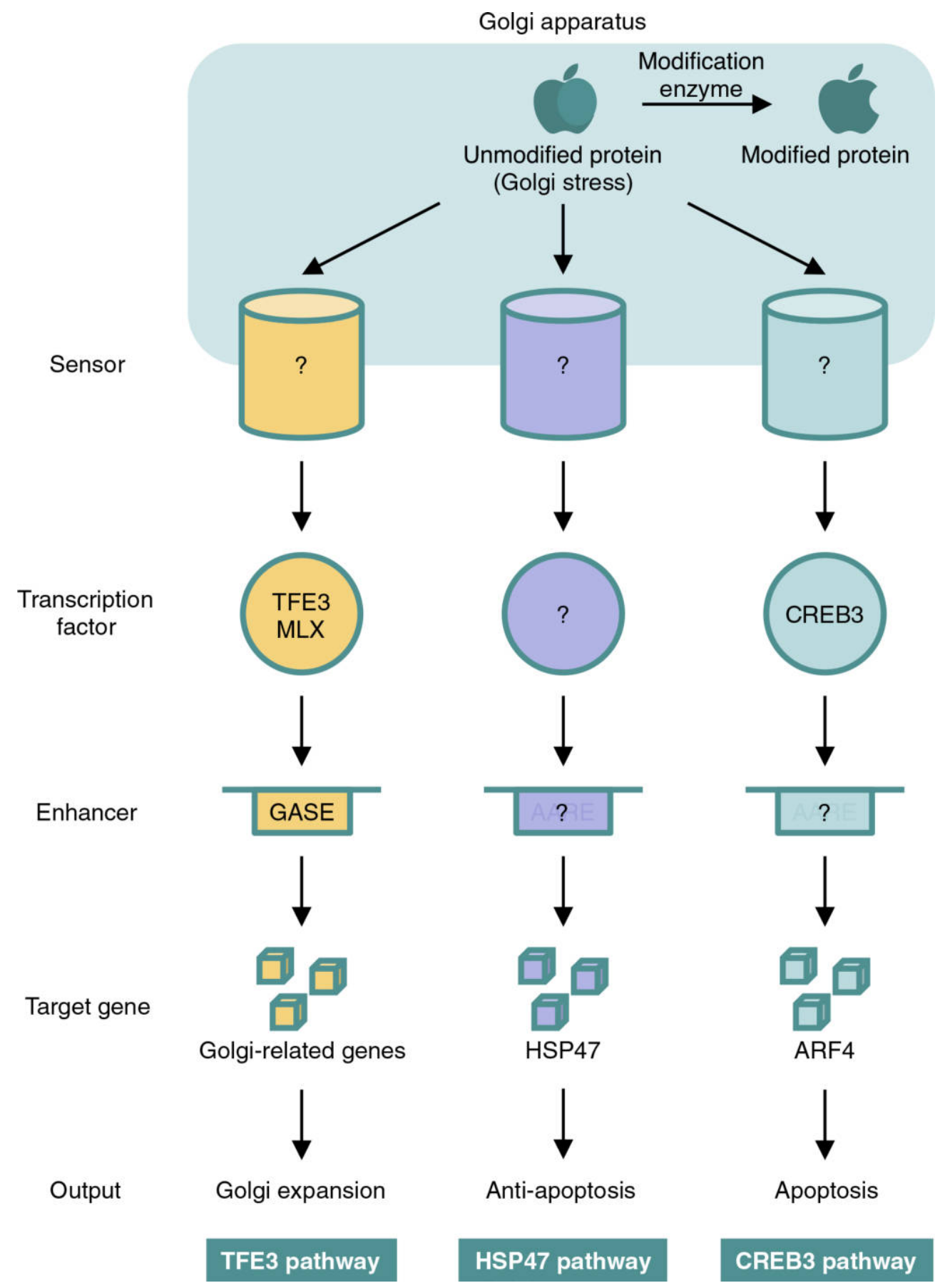

Fig. 3. The mechanism of the mammalian Golgi stress response. Secretory and membrane proteins receive post-translational modifications in the Golgi, including glycosylation, sulfation, and phosphorylation. When the synthesis of secretory and membrane proteins exceeds the capacity of the Golgi, most of them cannot be modified properly and cells have to increase their expression of modification enzymes. Although the molecular nature of Golgi stress has not been clarified yet, it is possible that sensors of Golgi stress detect the accumulation of proteins that are not properly modified. Then, sensors activate downstream transcription factors, leading to the transcriptional induction of Golgi-related genes. The figure shows three well-known pathways (the TFE3, HSP47, and CREB3 pathways), and it is highly possible that the mammalian Golgi stress response contains more diverse response pathways. 
reduced activity of ER chaperones by reducing $\mathrm{Ca}^{2+}$ concentrations in the ER, leading to insufficiency of folding function of the ER (ER stress) and activation of the ER stress response.

Similarly, one of Golgi stress inducers for the TFE3 pathway is monensin, an ionophore that antiports $\mathrm{Na}^{+}$and $\mathrm{H}^{+}$(Dinter and Berger, 1998). Monensin was originally developed as an antibiotic isolated from Streptomyces cinnamonensis, and is widely used in animal feeds. The Golgi apparatus is relatively acidic organelle $(\mathrm{pH} 6.58$ in the trans-Golgi), and most Golgi-localized proteins require an acidic environment for their maximum activity. Thus, upon monensin treatment, the $\mathrm{pH}$ of the Golgi becomes neutralized and the activity of Golgi proteins severely reduced, resulting in insufficiency in Golgi functions such as glycosylation and vesicular transport (Golgi stress). Although the lysosome and mitochondrial intermembrane space are also acidic compartments and could be affected by monensin treatment, monensin treatment hardly affected the expression of lysosomal and mitochondrial genes (Oku et al., 2011), probably as a result of differences in lipid content between the Golgi and lysosomal/mitochondrial membrane (Dinter and Berger, 1998). Upon monensin treatment, the Golgi apparatus becomes swollen and remarkably expanded because of the influx of water and/or accumulation of secretory proteins (Dinter and Berger, 1998). Usually, the Golgi cannot be observed by phase-contrast microscopy, but upon monensin treatment the Golgi swells so that it can be clearly detected (Oku et al., 2011). Nigericin, another ionophore that antiports $\mathrm{K}^{+}$and $\mathrm{H}^{+}$, is also used as a Golgi stress inducer in a working mechanism similar to monensin (Oku et al., 2011).

Another Golgi stress inducer of the TFE3 pathway is suppression of SLC35A1 expression by RNA interference (Taniguchi et al., 2015). SLC35A1 is a transporter that imports CMP-sialic acid from the cytosol to the Golgi. Because CMP-sialic acids are used for sialylation of proteins in the Golgi, sialic acid modification in the Golgi was blocked when SLC35A1 expression was knocked down (Xu et al., 2010), and then the TFE3 pathway was activated (Taniguchi et al., 2015), suggesting that insufficiency of glycosylation in the Golgi is important for activation of the TFE3 pathway.

The third method to activate the TFE3 pathway is ectopic expression of a dominant negative form of GCP60 (GCP60-DN) (Oku et al., 2011; Taniguchi et al., 2015). GCP60 is a Golgi structural protein associated with Giantin, a Golgi integral membrane protein (see below). Misumi and colleagues reported that overexpression of a dominant negative mutant of GCP60 caused disassembly of the Golgi structure and blocked protein transport from the ER to the Golgi (Sohda et al., 2001). Actually, overexpression of GCP60-DN activated the TFE3 pathway, possibly by disrupting Golgi function through Golgi disassembly (Taniguchi et al., 2015).

Inhibition of proteoglycan type glycosylation can also activate the TFE3 pathway. The first sugar conjugated to proteoglycan core proteins is always xylose (Miyata and Kitagawa, 2016; Nadanaka and Kitagawa, 2008). Both 4MU xyloside (4-methylumbelliferyl- $\beta$-D-xylopyranoside) and 4-NP xyloside (4-nitrophenyl- $\beta$-D-xylopyranoside) are chemically similar to xylose but they competitively inhibit conjugation of the second sugar (galactose) to the xylose residue conjugated to the proteoglycan core protein. Treatment with xylosides causes retention of proteoglycans in the Golgi, swelling and fragmentation of the Golgi apparatus (Kanwar et al., 1986), and activation of the TFE3 pathway (Taniguchi et al., 2015).

\section{(2) Target genes of the TFE3 pathway}

Table I shows target genes of the TFE3 pathway identified so far, and the expression of which is increased upon treatment with monensin, a Golgi stress inducer (Oku et al., 2011; Taniguchi et al., 2015). They include genes encoding post-translational modification enzymes localized in the Golgi, especially glycosylation enzymes, such as SIAT4A,

Table I. Golgi-Related genes, of Which EXPRession IS Induced by the TFE3 PathwaY

\begin{tabular}{ll}
\hline Gene name & \multicolumn{1}{c}{ Function } \\
\hline SIAT4A & CMP-N-acetylneuraminate-beta-galactosamide-alpha-2,3-sialyltransferase \\
SIAT10 & lactosamine alpha-2,3-sialyltransferase \\
FUT1 & galactoside 2-alpha-L-fucosyltransferase \\
B3GAT2 & beta-1,3-glucuronyltransferase \\
UAP1L1 & UDP-N-acetylglucosamine pyrophosphorylase beta-like protein \\
PCSK1 & proprotein convertase type 1 \\
GCP60 & Golgi structural protein \\
GM130 & Golgi matrix protein 130 kD, Golgi structural protein \\
Giantin & Golgi structural protein \\
STX3A & syntaxin 3A \\
WIPI49 & mannose-6-phosphate receptor recycling \\
RAB20 & small GTPase involved in endocytosis \\
\hline
\end{tabular}

Analyses with microarrays, next-generation DNA sequencers, and quantitative RT-PCR have been used to determine target genes of the TFE3 pathway. Details of the target genes are described in the text. 
SIAT10, FUT1, B3GAT2 and UAP1L1. SIAT4A (also called ST3GAL1), and SIAT10 (also called ST3GAL6) encoding the beta-galactoside alpha-2,3-sialyltransferase involved in glycosylation of N-glycans, proteoglycans, mucins, and glycosphingolipids. Additionally, FUT1 encodes the galactoside 2-L-fucosyltransferase involved in the glycosylation of N-glycans, O-glycans, glycosphingolipids, and the formation of $\mathrm{H}$ antigen in erythrocytes, of which mutations cause the H-Bombay blood group. B3GAT2 (also called GLCATS) is a glucuronyltransferase involved in the synthesis of the human natural killer-1 (HNK-1) carbohydrate epitope, which is important for neural development and function (Morita et al., 2008). UAP1L1 (UDP-Nacetylglucosamine pyrophosphorylase 1 like 1) is an enzyme involved in the synthesis of nucleotide sugars such as UDP-N-acetyl-D-galactosamine and UDP-N-acetyl-Dglucosamine, which are donor substrates for glycosyltransferases. PCSK1 (proprotein convertase subtilisin/kexin type 1) is a protease that processes protein and peptide precursor trafficking through regulated or constitutive branches of the secretory pathway. The substrates of PCSK1 include proglucagon and proinsulin.

Another set of target genes of the TFE3 pathway are Golgi structural proteins, including GCP60, Giantin, and GM130. GCP60 (also called ACBD3) is a Golgi resident protein involved in the maintenance of Golgi structure and function through its interaction with the integral membrane protein Giantin (Sohda et al., 2001), Golgin-160 (Sbodio and Machamer, 2007), PI4KB (Sasaki et al., 2012), protein phosphatase PPM1L (Shinoda et al., 2012), and Rab33 GTPase-activating proteins TBC1D22A and TBC1D22B (Greninger et al., 2013). Of note, the expression of GCP60 is markedly elevated in the striatum of Huntington disease (HD) patients, and enhanced levels of GCP60 elicited by endoplasmic reticulum, mitochondrial, and Golgi stresses may account for HD-associated neurodegeneration (Greninger et al., 2013). GM130 (also called Golgin-95) and Golgin-160 are cis-Golgi-localized members of Golgi family of coiled-coil tethering proteins and play important roles in the stacking of Golgi cisternae and in vesicular transport as a vesicle tethering factor (Nakamura, 2010). Giantin is a Golgi rim-localized member of the Golgin family and plays a role in vesicle tethering in conjunction with GM130 (Witkos and Lowe, 2015). Like Golgin-160, Giantin is associated with GCP60 in the Golgi.

The other target genes include components of vesicular transport (STX3A, WIPI49 and RAB20). STX3A is member of the syntaxin family and is important in establishing and maintaining polarity necessary for protein trafficking as well as in vesicle fusion and apical exocytosis (Vogel et al., 2015). Most STX3A is localized to the apical plasma membrane, whereas a significant proportion of syntaxin 3 $(25 \%)$ was detected in subcellular fractions containing transport vesicles (Fujita et al., 1998). WIPI49 (also called WIPI1 and ATG18) is a WD40 repeat protein that interacts with phosphoinositides and is localized to both trans-Golgi and endosomal membranes, where it regulates membrane traffic (Jeffries et al., 2004). RAB20 is a small GTPase localized in the Golgi and transport vesicles, and it is involved in membrane traffic such as phagocytosis (Egami and Araki, 2012).

Notably, expression of these target genes of the Golgi stress response is not induced by ER stress inducers, such as thapsigargin and tunicamycin, whereas expression of target genes of the ER stress response such as $\mathrm{BiP}$ is not induced by monensin treatment. Moreover, the ATF6 and IRE1 pathways of the ER stress response are not activated by monensin treatment (Oku et al., 2011). These observations suggest that the Golgi stress response is distinct from the ER stress response.

\section{(3) Enhancer element regulating the TFE3 pathway}

From the analysis of promoters of human SIAT4A and GCP60 genes, the enhancer element that regulates transcriptional induction of these target genes by Golgi stress was identified and named the Golgi apparatus stress responsive element (GASE), of which consensus nucleotide sequence was ACGTGGC (Oku et al., 2011). Moreover, GASE sequences are found in genes encoding murine and fish homologs, indicating that GASE has been conserved during the evolution of vertebrates.

\section{(4) Transcription factors regulating the TFE3 pathway}

TFE3 and MLX were identified as transcription factors regulating the TFE3 pathway (Taniguchi et al., 2015, 2016). Both TFE3 and MLX contain the basic helix-loop-helix leucine zipper (bHLH-ZIP) motif. The basic region is the DNA-binding domain, whereas the HLH-ZIP domain is the dimerization domain. Importantly, TFE3 has a strong transcriptional activation domain, whereas MLX does not. In normal growth conditions, TFE3 is phosphorylated at Ser108 and anchored in the cytoplasm as a dormant form (Fig. 4). Upon Golgi stress, TFE3 is dephosphorylated at Ser108 in the cytoplasm, translocates into the nucleus, binds to GASE, and activates the transcription of target genes. Neither kinases nor phosphatases responsible for TFE3 regulation have been identified yet. Similarly, MLX is retained in the cytoplasm in the absence of Golgi stress, whereas MLX translocates into the nucleus in response to Golgi stress. In the nucleus, MLX competes with TFE3 for GASE binding and reduces transcriptional induction by TFE3. Thus, TFE3 is a strong transcriptional activator, whereas MLX is a weak activator or attenuator of the TFE3 pathway. This is similar to the case of the ATF6 pathway of the ER stress response. The ATF6 pathway is regulated by two transcription factors, pATF $6 \alpha(\mathrm{N})$ and $\operatorname{pATF} 6 \beta(\mathrm{N})$, which are strong and weak activators similar to TFE3 and MLX, respectively.

TFE3 was first identified as a transcription factor binding to enhancer elements called $\mu \mathrm{E} 3$ and USF, of which the 


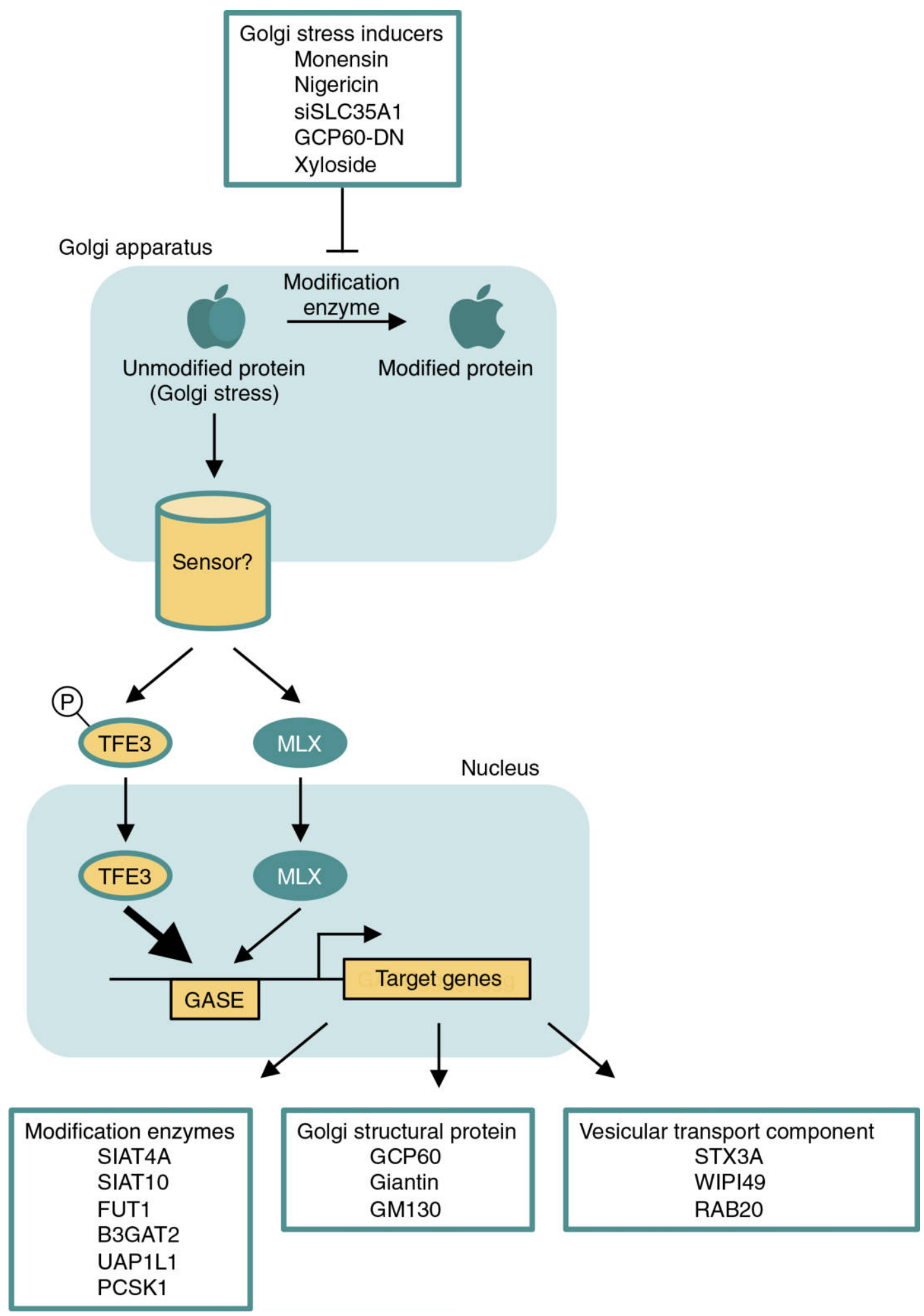

Fig. 4. The molecular mechanism of the TFE 3 pathway. To induce Golgi stress in experimental systems, various inhibitors of modification enzymes can be used, such as monensin and siRNAs for CMP-sialic acid transporter (siSLC35A1). The sensor molecule for the TFE3 pathway has not been identified yet. Upon Golgi stress, TFE3 and MLX translocate from the cytosol to the nucleus and regulate the transcription of Golgi-related genes through binding to GASE. TFE3 is a strong activator, while MLX seems to be a weak activator or a suppressor. 
consensus sequences are CAGGTGGC and CACGTGGC, respectively (Beckmann et al., 1990; Lenardo et al., 1987), and they are very similar to the consensus for GASE. TFE3 belongs to a MITF subfamily of the mammalian bHLH-ZIP transcription factors (Atchley and Fitch, 1997), which includes MITF, TFEB, TFEC, and TFE3. MITF is a transcription factor important for the biosynthesis of the melanosome and eye pigment (Steingrimsson et al., 2004), whereas TFEB is a key regulator of the lysosome stress response (Sardiello et al., 2009). The function of TFEC is still controversial. Because TFE3 regulates Golgi biogenesis, it is highly possible that MITF family transcription factors are important regulators specialized for organelle autoregulation.

MLX was first identified as a protein that binds to the transcription factor Mad (Meroni et al., 2000). MLX can form a heterodimer with Mondo-A and ChREBP/Mondo-B and upregulates transcription involved in lipogenic genes in response to glucose (Billin and Ayer, 2006). In normal growth conditions, ChREBP is phosphorylated and anchored in the cytoplasm as a dormant form, whereas ChREBP is dephosphorylated upon glucose signaling and translocates into the nucleus to upregulate transcription (Uyeda and Repa, 2006), which is quite similar to the regulation of TFE3. Thus, MLX seems to be a partner protein of various bHLH-ZIP transcription factors, including Mad, Mondo-A, ChREBP, and TFE3.

\section{(5) Sensors for Golgi stress and the sensing mechanism}

Sensor molecules of the TFE3 pathway and the sensing mechanism have not been identified yet. In the case of the ER stress response, unfolded proteins accumulated in the ER due to the insufficiency of ER function (protein folding function). It is believed that unfolded proteins bind directly to the MHC-like cleft of IRE1 to induce oligomerization and activation of IRE1 (Credle et al., 2005; Kimata et al., 2007). However, ER chaperones such as BiP are also involved in sensing ER stress. In normal growth conditions, BiP binds to IRE1 and ATF6 and keeps them inactive, whereas upon ER stress BiP is sequestered by unfolded proteins, resulting in the activation of IRE1 and ATF6 (Bertolotti et al., 2000; Okamura et al., 2000; Shen et al., 2002). In the case of the Golgi stress response, it is possible that Golgi stress causes accumulation of secretory glycoproteins that are not properly glycosylated due to insufficiency of glycosylation enzymes, resulting in activation of the TFE3 pathway. One possibility is that improperly glycosylated proteins bind directly to and activate the sensor for Golgi stress. Another scenario is that glycosylation enzymes bind and inactivate sensor molecules in normal growth conditions, and the enzymes are sequestered to improperly glycosylated proteins upon Golgi stress, resulting in activation of the sensors.

\section{HSP47 pathway}

The second pathway of the mammalian Golgi stress response is the HSP47 pathway, which was elucidated by Tohyama and colleagues (Miyata et al., 2013) (Fig. 3). The HSP47 pathway induces the expression of HSP47 in response to Golgi stress in order to prevent Golgi stressinduced apoptosis. Benzyl 2-acetamido-2-deoxy- $\alpha$-dgalactopyranoside (BG) was used to activate the HSP47 pathway. Mucin-type O-glycosylation occurs in the Golgi and the first sugar conjugated to mucin core proteins is GALNAc. Because BG is structurally similar to GALNAc, most second sugars are conjugated to $B G$ instead of GALNAc residues conjugated to mucin core proteins if cells are treated with $\mathrm{BG}$, resulting in competitive inhibition of mucin type O-glycosylation. To identify proteins in which their expression is induced by BG treatment, Colo 205 cells (human colorectal cancer cell line) were treated with BG and whole cell lysates were subjected to 2DPAGE, leading to the identification of HSP47. Quantitative real-time PCR experiments revealed that transcription of the human HSP47 gene was induced upon BG treatment, suggesting that the regulation of HSP47 expression is controlled at the transcriptional level. HSP47 is an ERlocalized molecular chaperone specialized for folding of collagens identified by Dr. Kazuhiro Nagata (Nagata et al., 1988), and important for proline hydroxylation as well as formation of triple helix of collagen molecules. Although HSP47 is an ER chaperone, its expression is not induced by ER stress inducers such as tunicamycin or thapsigargin but is increased by heat stress. Of note, HSP47 expression is induced by BG as well as monensin treatment (Miyata et al., 2013), suggesting its close link to Golgi function, and although HSP47 is constitutively localized in the ER, it does not translocate to the Golgi upon BG treatment.

When expression of HSP47 was suppressed by RNA interference, BG treatment resulted in expansion and fragmentation of the Golgi apparatus as well as activation of caspase-2 and apoptosis (Miyata et al., 2013). On the contrary, when HSP47 was overexpressed, BG-induced apoptosis was attenuated. These observations suggested that HSP47 is induced by the HSP47 pathway of the Golgi stress response in order to suppress Golgi stress-induced apoptosis. Because expression levels and localization of type IV collagen were not affected by BG treatment, this suggested that collagens are not involved in Golgi-stress induced apoptosis.

Deficiency of mucin type O-glycosylation occurs in the Golgi apparatus, whereas HSP47 functions in the ER, and it is unclear how HSP47 can suppress Golgi stress induced by BG treatment. Because collagens receive mucin type Oglycosylation (Taga et al., 2012), it is possible that a small amount of HSP47 transiently translocates to the Golgi, where HSP47 assists mucin type O-glycosylation of collagens. The link between HSP47 and the Golgi as well as the 
molecular mechanism of the HSP47 pathway are important issues for future analyses.

\section{CREB3 pathway}

The third pathway of the mammalian Golgi stress response is the CREB3 pathway, which was reported by Sabatini and colleagues (Reiling et al., 2013) (Fig. 4). The CREB3 pathway induces apoptosis in response to Golgi stress through transcriptional induction of ARF4. Brefeldin A (BFA) is used to activate the CREB3 pathway. BFA was identified as an anti-virus reagent and it inhibits small $G$ proteins such as ARFs. It was well known that BFA induces apoptosis, and Sabatini and colleagues tried to identify proteins involved in BFA-induced apoptosis through insertional mutagenesis using KBM cells (a human chronic myeloid leukemia cell line, which is near haploid). They isolated mutated KBM cells that were resistant to BFA-induced apoptosis and revealed that the gene responsible is ARF4.

When the expression of ARF4 was suppressed by RNA interference, apoptosis induced by Golgi stress inducers such as BFA, monensin, Gogicide A (an inhibitor of GBF1 (a GEF for ARF), and Exo1 (an inhibitor of ARF) was remarkably reduced. Of note, the transcription of ARF4 was induced by the transcription factor CREB3/Luman in response to BFA treatment. CREB3 belongs to a ATF6 family and is a transmembrane protein localized in the ER. Upon treatment with Golgi stress inducers, CREB3 was transported from the ER to Golgi, cleaved by S1P and S2P proteases, and activated transcription of ARF4, resulting in Golgi stress-induced apoptosis. It was reported that CREB3 is activated by ER stress (Liang et al., 2006), and that the Golgi membrane, as well as S1P and S2P proteases, is absorbed by the ER membrane upon BFA treatment, resulting in the cleavage of ATF6 family proteins (Shen and Prywes, 2004), including CREB3. Thus, it would be a very interesting project to reveal how Golgi stress activates CREB3.

\section{Conclusion}

Currently, three response pathways, namely the TFE3, HSP47, and CREB3 pathways, have been identified in the mammalian Golgi stress response. However, the pathways of the Golgi stress response should be much more diverse, because the structure and function of the Golgi is much more complicated than that of the ER. Revealing the molecular mechanism of the Golgi stress response should contribute greatly to cell biology, and could lead to the identification of diseases related to the Golgi stress response.

Acknowledgments. We thank Ms. Mikiko Ochiai for secretarial assistance. This work was supported by MEXT and JSPS KAKENHI (Grant
Number 25650070, 16K07356 and 15J05492) and a Grant-in-Aid for Scientific Research on Innovative Areas of MEXT (No. 23110007).

\section{References}

Atchley, W.R. and Fitch, W.M. 1997. A natural classification of the basic helix-loop-helix class of transcription factors. Proc. Natl. Acad. Sci. USA, 94: 5172-5176.

Beckmann, H., Su, L.K., and Kadesch, T. 1990. TFE3: a helix-loop-helix protein that activates transcription through the immunoglobulin enhancer muE3 motif. Genes Dev., 4: 167-179.

Berger, E.G. and Roth, J. 1997. The Golgi Apparatus. Birkheuser Verlag, Basel.

Bertolotti, A., Zhang, Y., Hendershot, L.M., Harding, H.P., and Ron, D. 2000. Dynamic interaction of BiP and ER stress transducers in the unfolded-protein response. Nat. Cell Biol., 2: 326-332.

Billin, A.N. and Ayer, D.E. 2006. The Mlx network: evidence for a parallel Max-like transcriptional network that regulates energy metabolism. Curr. Top. Microbiol. Immunol., 302: 255-278.

Chakravarthy, M.V., Lodhi, I.J., Yin, L., Malapaka, R.R., Xu, H.E., Turk, J., and Semenkovich, C.F. 2009. Identification of a physiologically relevant endogenous ligand for PPARalpha in liver. Cell, 138: 476-488.

Credle, J.J., Finer-Moore, J.S., Papa, F.R., Stroud, R.M., and Walter, P. 2005. On the mechanism of sensing unfolded protein in the endoplasmic reticulum. Proc. Natl. Acad. Sci. USA, 102: 18773-18784.

Dinter, A. and Berger, E.G. 1998. Golgi-disturbing agents. Histochem Cell Biol., 109: 571-590.

Egami, Y. and Araki, N. 2012. Rab20 regulates phagosome maturation in RAW264 macrophages during Fc gamma receptor-mediated phagocytosis. PLoS One, 7: e35663.

Fujita, H., Tuma, P.L., Finnegan, C.M., Locco, L., and Hubbard, A.L. 1998. Endogenous syntaxins 2, 3 and 4 exhibit distinct but overlapping patterns of expression at the hepatocyte plasma membrane. Biochem. J., 329(Pt 3): 527-538.

Gardner, B.M., Pincus, D., Gotthardt, K., Gallagher, C.M., and Walter, P. 2013. Endoplasmic reticulum stress sensing in the unfolded protein response. Cold Spring Harb. Perspect. Biol., 5: a013169.

Greninger, A.L., Knudsen, G.M., Betegon, M., Burlingame, A.L., and DeRisi, J.L. 2013. ACBD3 interaction with TBC1 domain 22 protein is differentially affected by enteroviral and kobuviral $3 \mathrm{~A}$ protein binding. MBio, 4: e00098-13.

Jeffries, T.R., Dove, S.K., Michell, R.H., and Parker, P.J. 2004. PtdInsspecific MPR pathway association of a novel WD40 repeat protein, WIPI49. Mol. Biol. Cell, 15: 2652-2663.

Kanwar, Y.S., Rosenzweig, L.J., and Jakubowski, M.L. 1986. Xylosylated-proteoglycan-induced Golgi alterations. Proc. Natl. Acad. Sci. USA, 83: 6499-6503.

Kimata, Y., Ishiwata-Kimata, Y., Ito, T., Hirata, A., Suzuki, T., Oikawa, D., Takeuchi, M., and Kohno, K. 2007. Two regulatory steps of ERstress sensor Ire1 involving its cluster formation and interaction with unfolded proteins. J. Cell Biol., 179: 75-86.

Kimata, Y. and Kohno, K. 2011. Endoplasmic reticulum stress-sensing mechanisms in yeast and mammalian cells. Curr. Opin. Cell Biol., 23: 135-142.

Lenardo, M., Pierce, J.W., and Baltimore, D. 1987. Protein-binding sites in Ig gene enhancers determine transcriptional activity and inducibility. Science, 236: 1573-1577.

Liang, G., Audas, T.E., Li, Y., Cockram, G.P., Dean, J.D., Martyn, A.C., Kokame, K., and Lu, R. 2006. Luman/CREB3 induces transcription of the endoplasmic reticulum (ER) stress response protein Herp through an ER stress response element. Mol. Cell. Biol., 26: 7999-8010.

Lin, Y.F. and Haynes, C.M. 2016. Metabolism and the UPR(mt). Mol. Cell, 61: 677-682.

Meroni, G., Cairo, S., Merla, G., Messali, S., Brent, R., Ballabio, A., and 
Reymond, A. 2000. Mlx, a new Max-like bHLHZip family member: the center stage of a novel transcription factors regulatory pathway? Oncogene, 19: 3266-3277.

Miyata, S., Mizuno, T., Koyama, Y., Katayama, T., and Tohyama, M. 2013. The endoplasmic reticulum-resident chaperone heat shock protein 47 protects the Golgi apparatus from the effects of O-glycosylation inhibition. PLoS One, 8: e69732.

Miyata, S. and Kitagawa, H. 2016. Chondroitin sulfate and neuronal disorders. Front. Biosci. (Landmark Ed), 21: 1330-1340.

Mori, K. 2015. The unfolded protein response: the dawn of a new field. Proc. Jpn. Acad. Ser. B Phys. Biol. Sci., 91: 469-480.

Morita, I., Kizuka, Y., Kakuda, S., and Oka, S. 2008. Expression and function of the HNK-1 carbohydrate. J. Biochem., 143: 719-724.

Nadanaka, S. and Kitagawa, H. 2008. Heparan sulphate biosynthesis and disease. J. Biochem., 144: 7-14.

Nagata, K., Hirayoshi, K., Obara, M., Saga, S., and Yamada, K.M. 1988. Biosynthesis of a novel transformation-sensitive heat-shock protein that binds to collagen. Regulation by mRNA levels and in vitro synthesis of a functional precursor. J. Biol Chem, 263: 8344-8349.

Nakamura, N. 2010. Emerging new roles of GM130, a cis-Golgi matrix protein, in higher order cell functions. J. Pharmacol. Sci., 112: 255264.

Napolitano, G. and Ballabio, A. 2016. TFEB at a glance. J. Cell Sci., 129: $2475-2481$.

Okamura, K., Kimata, Y., Higashio, H., Tsuru, A., and Kohno, K. 2000. Dissociation of Kar2p/BiP from an ER sensory molecule, Ire1p, triggers the unfolded protein response in yeast. Biochem. Biophys. Res. Commun., 279: 445-450.

Oku, M., Tanakura, S., Uemura, A., Sohda, M., Misumi, Y., Taniguchi, M., Wakabayashi, S., and Yoshida, H. 2011. Novel cis-acting element GASE regulates transcriptional induction by the Golgi stress response. Cell Struct. Funct., 36: 1-12.

Reiling, J.H., Olive, A.J., Sanyal, S., Carette, J.E., Brummelkamp, T.R., Ploegh, H.L., Starnbach, M.N., and Sabatini, D.M. 2013. A CREB3ARF4 signalling pathway mediates the response to Golgi stress and susceptibility to pathogens. Nat. Cell Biol., 15: 1473-1485.

Sardiello, M., Palmieri, M., di Ronza, A., Medina, D.L., Valenza, M., Gennarino, V.A., Di Malta, C., Donaudy, F., Embrione, V., Polishchuk, R.S., Banfi, S., Parenti, G., Cattaneo, E., and Ballabio, A. 2009. A gene network regulating lysosomal biogenesis and function. Science, 325: 473-477.

Sasaki, J., Ishikawa, K., Arita, M., and Taniguchi, K. 2012. ACBD3mediated recruitment of PI4KB to picornavirus RNA replication sites. EMBO J., 31: 754-766.

Sasaki, K. and Yoshida, H. 2015. Organelle autoregulation-stress responses in the ER, Golgi, mitochondria and lysosome. J. Biochem., 157: $185-195$.

Sbodio, J.I. and Machamer, C.E. 2007. Identification of a redox-sensitive cysteine in GCP60 that regulates its interaction with golgin-160. J. Biol.
Chem., 282: 29874-29881.

Shen, J., Chen, X., Hendershot, L., and Prywes, R. 2002. ER stress regulation of ATF6 localization by dissociation of BiP/GRP78 binding and unmasking of Golgi localization signals. Dev. Cell, 3: 99-111.

Shen, J. and Prywes, R. 2004. Dependence of site-2 protease cleavage of ATF6 on prior site-1 protease digestion is determined by the size of the luminal domain of ATF6. J. Biol. Chem., 279: 43046-43051.

Shinoda, Y., Fujita, K., Saito, S., Matsui, H., Kanto, Y., Nagaura, Y., Fukunaga, K., Tamura, S., and Kobayashi, T. 2012. Acyl-CoA binding domain containing 3 (ACBD3) recruits the protein phosphatase PPM1L to ER-Golgi membrane contact sites. FEBS Lett., 586: 3024-3029.

Sohda, M., Misumi, Y., Yamamoto, A., Yano, A., Nakamura, N., and Ikehara, Y. 2001. Identification and characterization of a novel Golgi protein, GCP60, that interacts with the integral membrane protein giantin. J. Biol. Chem., 276: 45298-45306.

Steingrimsson, E., Copeland, N.G., and Jenkins, N.A. 2004. Melanocytes and the microphthalmia transcription factor network. Annu. Rev. Genet., 38: 365-411.

Taga, Y., Kusubata, M., Ogawa-Goto, K., and Hattori, S. 2012. Development of a novel method for analyzing collagen O-glycosylations by hydrazide chemistry. Mol. Cell. Proteomics, 11: M111 010397.

Taniguchi, M., Nadanaka, S., Tanakura, S., Sawaguchi, S., Midori, S., Kawai, Y., Yamaguchi, S., Shimada, Y., Nakamura, Y., Matsumura, Y., Fujita, N., Araki, N., Yamamoto, M., Oku, M., Wakabayashi, S., Kitagawa, H., and Yoshida, H. 2015. TFE3 Is a bHLH-ZIP-type Transcription Factor that Regulates the Mammalian Golgi Stress Response. Cell Struct. Funct., 40: 13-30.

Taniguchi, M., Sasaki-Osugi, K., Oku, M., Sawaguchi, S., Tanakura, S., Kawai, Y., Wakabayashi, S., and Yoshida, H. 2016. MLX Is a Transcriptional Repressor of the Mammalian Golgi Stress Response. Cell Struct. Funct., 41: 93-104.

Uyeda, K. and Repa, J.J. 2006. Carbohydrate response element binding protein, ChREBP, a transcription factor coupling hepatic glucose utilization and lipid synthesis. Cell Metabolism, 4: 107-110.

Vogel, G.F., Klee, K.M., Janecke, A.R., Muller, T., Hess, M.W., and Huber, L.A. 2015. Cargo-selective apical exocytosis in epithelial cells is conducted by Myo5B, Slp4a, Vamp7, and Syntaxin 3. J. Cell Biol., 211: 587-604.

Volmer, R. and Ron, D. 2015. Lipid-dependent regulation of the unfolded protein response. Curr. Opin. Cell Biol., 33: 67-73.

Witkos, T.M. and Lowe, M. 2015. The Golgin Family of Coiled-Coil Tethering Proteins. Front. Cell Dev. Biol., 3: 86.

Xu, Y.X., Liu, L., Caffaro, C.E., and Hirschberg, C.B. 2010. Inhibition of Golgi apparatus glycosylation causes endoplasmic reticulum stress and decreased protein synthesis. J. Biol. Chem., 285: 24600-24608.

Yoshida, H. 2007. ER stress and diseases. FEBS J., 274: 630-658.

(Received for publication, December 29, 2016, accepted, January 19, 2017 and published online, February 7, 2017) 